\title{
Ubrogepant in the Acute Management of Migraine: A Narrative Review
}

\author{
Chia-Chun Chiang (D) \\ Juliana $\mathrm{H}$ VanderPluym ${ }^{2}$ \\ 'Department of Neurology, Mayo Clinic, \\ Rochester, MN, USA; ${ }^{2}$ Department of \\ Neurology, Mayo Clinic, Scottsdale, \\ AZ, USA
}

\begin{abstract}
Ubrogepant is a small-molecule calcitonin gene-related peptide (CGRP) receptor antagonist that received Food and Drug Administration (FDA) approval for the acute treatment of migraine with and without aura in adults. The ACHIEVE I and ACHIEVE II Phase III clinical trials showed that ubrogepant was superior to placebo for pain freedom and freedom of the most bothersome migraine-associated symptom at 2 hours after medication intake. The 52-week open label extension of the Phase III trials demonstrated safety of ubrogepant. A real-world study conducted at a tertiary headache center also confirmed the efficacy and safety of ubrogepant. Adverse event rates were higher in the real-world population. Studies are needed to evaluate its long-term efficacy and safety, especially in the setting of co-administration with other CGRP modulating therapies such as the CGRP monoclonal antibodies.
\end{abstract}

Keywords: ubrogepant, CGRP, migraine, Phase III trials, real-world

\section{Introduction}

Ubrogepant, a small-molecule calcitonin gene-related peptide (CGRP) receptor antagonist, was the first in the class of oral CGRP receptor antagonists that received approval by the Food and Drug Administration (FDA) on December 23, 2019 for the acute treatment of migraine with or without aura in adults. Two Phase III trials, the ACHIEVE I and ACHIEVE II, established efficacy and safety of ubrogepant. ${ }^{1,2}$ Guidelines for acute treatment of migraine require updates to reflect the place for this new therapy. The guideline updates will need to consider ubrogepant's efficacy, side effect and interaction profile, and cost and insurance coverage compared to other therapies. The price of ubrogepant is much higher than other acute treatments for migraine such as triptans. For example, according to GoodRx.com searched on $1 / 25 / 2021$, a monthly supply of 9 tablets of sumatriptan costs less than \$20 USD while a monthly supply of ubrogepant of 10 tablets costs around \$1000 USD. Currently, most insurance requires patients to have prior unsuccessful treatment response or intolerance to at least 2 or 3 different triptans before covering ubrogepant. In this review, we provide an overview of the pharmacology, efficacy and safety data of ubrogepant from clinical trials and real-world studies.

\section{CGRP-Targeted Therapies}

CGRP is a neuropeptide that plays a pivotal role in the pathophysiology of migraine. Calcitonin (CT), amylin (AMY) and adrenomedullin (AM) are other peptides that also belong to the calcitonin family. The CGRP receptor is made up of a calcitonin
Correspondence: Juliana $\mathrm{H}$ VanderPluym Email vanderpluym.juliana@mayo.edu 
receptor (CTR)-like receptor (CLR) and receptor activity modifying protein 1 ( RAMP $\left._{1}\right)$. The AMY receptors are a complex of the CTR and RAMP ${ }_{1}, \mathrm{RAMP}_{2}$, or RAMP . $_{3}$ Studies have shown that ubrogepant also demonstrated antagonist activity on the AMY receptors, especially $\mathrm{AMY}_{1}$; although its potency on CGRP receptors was much stronger than AMY receptors. ${ }^{3,4}$ It is believed that CGRP promotes nociceptive transmission, modulates vasodilation and causes neurogenic inflammation. ${ }^{4}$ The main types of CGRP function-blocking modalities include monoclonal antibodies (mAbs) targeting the CGRP ligand or receptor, and small molecule antagonists (gepants) targeting the CGRP receptor. There are four CGRP monoclonal antibodies that are available in the United States as migraine preventive medications- erenumab, fremanezumab, galcanezumab, and eptinezumab. All are monthly or quarterly subcutaneous injections, except eptinezumab which is given intravenously every 3 months. Currently, two gepants have received FDA approval for the acute treatment of migraine in adults, ubrogepant and rimegepant. They are oral tablets or oral disintegrating tablets to be taken as needed for migraine. A recent study showed that rimegepant, when given every other day, was effective to reduce the mean monthly migraine days compared to placebo. ${ }^{5}$ At the time that this manuscript was written rimegepant remained FDA approved only for the indication of acute treatment of migraine. Several other gepants are being studied for the acute and preventive treatment of migraine. ${ }^{6}$

\section{Pharmacokinetic Data of Ubrogepant}

Ubrogepant has a peak plasma concentration at 1.5 hours after ingestion and is metabolized by cytochrome P450 3A4 (CYP3A4). The elimination half-life of ubrogepant is approximately $5-7$ hours. ${ }^{7}$ Ubrogepant is excreted through the biliary, fecal (major), and renal (minor) routes. Two dosages, $50 \mathrm{mg}$ and $100 \mathrm{mg}$ of ubrogepant are available for clinical use and can be repeated once after $\geq 2$ hours if needed. In general, the maximum dose is $200 \mathrm{mg}$ per 24 hours; however, dosage adjustment is recommended for patients using concomitant CYP3A4 inhibitors, those with renal impairment or hepatic impairment as outlined in Table 1 . The safety of treating $>8$ migraines/month has not been established. ${ }^{7,8}$
Table I Recommended Dosage of Ubrogepant in Adults with Concomitant Therapy, Hepatic or Renal Impairment ${ }^{4,6}$

\begin{tabular}{|c|c|}
\hline Condition & $\begin{array}{l}\text { Recommended Dosage } \\
\text { Adjustment }\end{array}$ \\
\hline Regular & $\begin{array}{l}\text { Initial: } 50 \text { to } 100 \mathrm{mg} \text { as a single dose; may } \\
\text { repeat once after } \geq 2 \text { hours. Maximum } \\
\text { dose: } 200 \mathrm{mg} \text { per } 24 \text { hours. }\end{array}$ \\
\hline \multicolumn{2}{|l|}{ Concomitant therapy } \\
\hline $\begin{array}{l}\text { Strong CYP3A4 inhibitor } \\
\text { (eg, ketoconazole, itraconazole, } \\
\text { clarithromycin) }\end{array}$ & Use is contraindicated \\
\hline $\begin{array}{l}\text { Moderate CYP3A4 inhibitor (eg, } \\
\text { cyclosporine, ciprofloxacin, } \\
\text { fluconazole, fluvoxamine, grapefruit } \\
\text { juice, verapamil) }\end{array}$ & $\begin{array}{l}50 \mathrm{mg} \text { as a single dose and } \\
\text { avoid second dose within } 24 \text { hours. } \\
\text { Maximum dose: } 50 \mathrm{mg} \text { per } 24 \text { hours. }\end{array}$ \\
\hline Weak CYP3A4 inhibitor & $\begin{array}{l}50 \mathrm{mg} \text { as a single dose, may repeat once } \\
\text { after } \geq 2 \text { hours. } \\
\text { Maximum dose: } 100 \mathrm{mg} \text { per } 24 \text { hours. }\end{array}$ \\
\hline $\begin{array}{l}\text { Strong CYP3A4 inducer } \\
\text { (eg phenytoin, barbiturates, rifampin, } \\
\text { St. John's wort) }\end{array}$ & Avoid concurrent use \\
\hline \multicolumn{2}{|l|}{ Renal Impairment } \\
\hline $\mathrm{CrCl} \geq 30 \mathrm{~mL} /$ minute & No dosage adjustment necessary \\
\hline $\mathrm{CrCl} 15$ to $29 \mathrm{~mL} /$ minute & $\begin{array}{l}50 \mathrm{mg} \text { as a single dose; may repeat } \\
\text { once after } \geq 2 \text { hours. } \\
\text { Maximum dose: } 100 \mathrm{mg} \text { per } 24 \text { hours. }\end{array}$ \\
\hline $\mathrm{CrCl}<15 \mathrm{~mL} /$ minute & Avoid use (has not been studied) \\
\hline \multicolumn{2}{|l|}{ Hepatic Impairment } \\
\hline $\begin{array}{l}\text { Mild to moderate impairment (Child- } \\
\text { Pugh class A, B) }\end{array}$ & No dosage adjustment necessary \\
\hline $\begin{array}{l}\text { Severe impairment (Child-Pugh } \\
\text { class C) }\end{array}$ & $\begin{array}{l}50 \mathrm{mg} \text { as a single dose, may repeat } \\
\text { once after } \geq 2 \text { hours. } \\
\text { Maximum dose: } 100 \mathrm{mg} \text { per } 24 \text { hours. }\end{array}$ \\
\hline
\end{tabular}

\section{Phase III Clinical Trials and Real-World Studies of Ubrogepant}

The ACHIEVE I and ACHIEVE II are two large, randomized, double-blind, placebo-controlled, parallel-group, single attack trials conducted at 89 and 99 centers, respectively, in the United States that established the efficacy and tolerability of ubrogepant. ${ }^{1,2}$ The designs of the two trials were very similar, but the dosage regimens were slightly 
different. Participants were randomly assigned in a $1: 1: 1$ ratio to receive placebo, ubrogepant $50 \mathrm{mg}$, or ubrogepant $100 \mathrm{mg}$ in ACHIEVE I, versus placebo, ubrogepant 25mg, or ubrogepant 50mg in ACHIEVE II. Randomization was stratified according to the participant's previous response to triptans and whether they were using preventive migraine medications.

For both ACHIEVE I and ACHIEVE II, participants were 18 to 75 years of age, had at least a 1-year history of migraine with or without aura, and had migraine onset before the age of 50 years. Participants had two to eight migraine attacks with moderate to severe head pain per month before screening, with headache freedom at least 48 hours between the migraine episodes. Of note, patients with a diagnosis of chronic migraine per the International Classification of Headache Disorders (ICHD) -3-beta, as well as patients who were taking acute migraine medications on 10 or more days per month were excluded. Patients who had received CGRP mAbs through clinical trials were also excluded. In ACHIEVE I, participants with moderate-to-severe cardiovascular risk factors, but not clinically significant cardiovascular or cerebrovascular diseases, were eligible. Participants were additionally excluded if they had abnormal liver function tests, defined as levels of alanine aminotransferase (ALT) or aspartate aminotransferase (AST) that were more than 1.5 times the upper normal limit, a total bilirubin level of more than $1.5 \mathrm{mg}$ per deciliter (26 $\mu \mathrm{mol}$ per liter), or a serum albumin level of less than $2.8 \mathrm{~g}$ per deciliter at screening. ${ }^{2}$ In ACHIEVE II, participants with clinically significant hematologic, endocrine, cardiovascular, cerebrovascular, pulmonary, renal, hepatic, gastrointestinal, or neurologic disease were excluded. ${ }^{1}$

Patients were asked to take one tablet of study medication within 4 hours of the onset of a migraine attack with moderate or severe head pain, and at least one of the following migraine-associated symptoms (photophobia, phonophobia or nausea). An optional second dose was allowed for the treatment of moderate or severe headache from 2 to 24 hours after the initial dose. For both trials, the co-primary efficacy end points were freedom from pain (from moderate to severe pain to no pain) at 2 hours after the initial dose of ubrogepant or placebo, and absence of the most bothersome symptom associated with migraine (photophobia, phonophobia and nausea) at 2 hours.

There are limitations to the design of the ACHIEVE I and ACHIEVE II trials. The results of the single-attack evaluation need to be confirmed by more long-term and multi-attack follow-up data. Furthermore, patients with a history of clinically significant cardiovascular diseases were excluded. More data is needed to evaluate the safety and efficacy of ubrogepant in this population. This is especially important as in the post-market setting de novo or worsening pre-existing hypertension has been reported following the use of erenumab, a monoclonal antibody, resulting in revision of its package insert. ${ }^{9}$ Erenumab and ubrogepant both target the CGRP receptor. In addition, patients were instructed to treat their migraine when the headache pain was moderate or severe. In clinical practice, patients are typically instructed to treat their migraine when pain is still mild, and it is anticipated that the gepants would have greater efficacy when treating mild pain.

Since ubrogepant became available for prescription in January 2020, we started to collect real-world patient experience data at our institution. The responses of a standardized questionnaire from 106 patients were analyzed and presented in a previous manuscript. ${ }^{10}$ Safety data from the open-label, 52-week extension phase of the ACHIEVE I and ACHIEVE II trials has also been published. ${ }^{11}$ The data from the 52-week open extension trial, as well as the real-world patient experience data complements and extends the results from the Phase III clinical trials and provides additional information to address the above limitations.

\section{Efficacy of Ubrogepant}

In ACHIEVE I, the intention-to-treat population included 1327 participants. The number of participants with freedom from migraine pain at 2 hours after the initial dose was 54 of $456(11.8 \%)$ in the placebo group, 81 of 422 (19.2\%; $\mathrm{p}=0.002$ for the comparison with placebo) in the 50 -mg ubrogepant group, and 95 of $448(21.2 \%$; $<<0.001)$ in the 100-mg ubrogepant group. Absence of the most bothersome migraine-associated symptom was reported in 126 of 454 participants $(27.8 \%)$ in the placebo group, in 162 of $420(38.6 \% ; \mathrm{p}=0.002)$ in the 50 -mg ubrogepant group, and in 169 of $448(37.7 \%$; $=0.002)$ in the $100-\mathrm{mg}$ ubrogepant group. The number of participants who had pain relief at 2 hours was 224 of $456(49.1 \%)$ in the placebo group, 256 of $422(60.7 \% ; p=0.002)$ in the 50 mg ubrogepant group, and 275 of $448(61.4 \% ; p=0.002)$ in the 100-mg ubrogepant group [Table 2]. The study concluded that both $50 \mathrm{mg}$ and $100 \mathrm{mg}$ of ubrogepant demonstrated significantly greater rates of pain freedom and freedom from the most bothersome migraine- 
Table 2 The Results of the Efficacy Primary Endpoints in ACHIEVE I and ACHIEVE II,2

\begin{tabular}{|c|c|c|c|}
\hline ACHIEVE I & Placebo & $50 \mathrm{mg}$ & $100 \mathrm{mg}$ \\
\hline$\%$ of patients with freedom from pain at 2 hours (moderate/severe pain to 0 pain) & $11.8 \%$ & $\begin{array}{l}19.2 \% \\
(p=0.002)\end{array}$ & $\begin{array}{l}21.2 \% \\
(p<0.001)\end{array}$ \\
\hline $\begin{array}{l}\% \text { of patients with absence of the most bothersome migraine-associated symptom (photophobia, } \\
\text { phonophobia, nausea) }\end{array}$ & $27.8 \%$ & $\begin{array}{l}38.6 \% \\
(p=0.002)\end{array}$ & $\begin{array}{l}37.7 \% \\
(p=0.002)\end{array}$ \\
\hline ACHIEVE II & Placebo & $25 \mathrm{mg}$ & $50 \mathrm{mg}$ \\
\hline$\%$ of patients with freedom from pain at 2 hours (moderate/severe pain to 0 pain) & $14.3 \%$ & $\begin{array}{l}20.7 \% \\
(p=0.03)\end{array}$ & $\begin{array}{l}21.8 \% \\
(p=0.01)\end{array}$ \\
\hline $\begin{array}{l}\% \text { of patients with absence of the most bothersome migraine-associated symptom (photophobia, } \\
\text { phonophobia, nausea) }\end{array}$ & $27.4 \%$ & $\begin{array}{l}34.1 \% \\
(p=0.07)\end{array}$ & $\begin{array}{l}38.9 \% \\
(p=0.01)\end{array}$ \\
\hline
\end{tabular}

associated symptom at two hours, compared with placebo. $^{2}$ In ACHIEVE II, a total of 1355 of the randomized 1465 participants $(92.5 \%)$ were evaluable for efficacy analyses. Pain freedom at 2 hours was reported by 65 of $456(14.3 \%)$ in the placebo group, 90 of $435(20.7 \%$; $\mathrm{p}=0.03$ ) in the ubrogepant 25-mg group, and 101 of 464 participants $(21.8 \%$; $=0.01)$ in the ubrogepant $50-\mathrm{mg}$ group, absence of the most bothersome associated symptom at 2 hours was reported by 125 of $456(27.4 \%)$ in the placebo group, 148 of $434(34.1 \% ; p=0.07)$ in the ubrogepant $25-\mathrm{mg}$ group, and 180 of 463 participants $(38.9 \% ; \mathrm{p}=0.01)$ in the ubrogepant $50-\mathrm{mg}$ group. The study concluded that ubrogepant $50 \mathrm{mg}$, but not $25 \mathrm{mg}$ dose, was significantly more effective than placebo regarding absence of the most bothersome migraine-associated symptom at 2 hours [Table 2]. ${ }^{1}$

The efficacy of ubrogepant was confirmed in a realworld patient experience study conducted in a tertiary headache center, where the majority of patients, $86.6 \%$, had chronic migraine, and had experienced lack of efficacy or intolerance to 7 (median, range $0-34$ ) preventive and 5 (median, range 0-32) acute migraine medications, and $77.5 \%$ patients had tried and found ineffective or had intolerance to at least two unique triptans. ${ }^{10}$ Based on the results of 106 survey responses, complete headache freedom (from mild/moderate/severe to no pain), and headache relief (from moderate/severe to mild/no pain or mild to no pain) for $\geq 75 \%$ of all treated attacks at 2 hours after taking ubrogepant was achieved in $19.0 \%$ and $47.6 \%$ patients, respectively. Additionally, a total of $31.1 \%$ patients in this group reported being "very satisfied" with ubrogepant, $38.7 \%$ patients reported being "somewhat satisfied" and 30.2\% patients reported "not at all satisfied" with ubrogepant. ${ }^{10}$

\section{Ubrogepant for the Treatment of Mild Head Pain}

The ACHIEVE I and ACHIEVE II Phase III clinical trials instructed patients to take ubrogepant when the pain was moderate to severe. The results of the open-label, 52-week extension phase of both trials reported the treatment efficacy when ubrogepant was used to treat mild headaches. It was reported that $39 \%$ and $43 \%$ of patients in the $50-\mathrm{mg}$ and 100-mg group, respectively, achieved pain freedom in attacks of mild pain, compared to $19 \%$ and $21 \%$, respectively, in those groups when treating attacks of moderate to severe pain $\left(\mathrm{P}<0.0001\right.$ for both). ${ }^{12}$

In the real-world study, 56 patients used ubrogepant while the pain was mild, and ubrogepant was able to abort mild headache attacks reliably for $\geq 75 \%$ of the attacks for $23(41.1 \%)$ patients, as opposed to achieving pain freedom for $\geq 75 \%$ of the attacks for $19.0 \%$ patients for all treated attacks, regardless of pain severity. ${ }^{10}$ This supports the efficacy of ubrogepant for mild headache attacks, and the general clinical recommendation to treat headache early when the pain is still mild for better treatment efficacy.

\section{Ubrogepant in Triptan Insufficient Responders}

A post-hoc analysis of ACHIEVE I and ACHIEVE II was conducted to assess the efficacy of ubrogepant for the acute treatment of migraine in triptan-insufficient responders. The results were presented in the American Headache Society Scientific meeting but at the time of 
this manuscript not yet published. In the Phase III trials, randomization was stratified according to the participant's previous response to triptans (history of a response, history of an insufficient response, or no history of receiving triptans). The authors reported that at 2, 4 and 8 hours post-initial dose of ubrogepant $50 \mathrm{mg}$, significantly more triptan-insufficient responders treated with ubrogepant reported being able to function normally versus those in the placebo group. Additionally, a greater proportion of triptan-insufficient responders were "satisfied" or "extremely satisfied" with ubrogepant $50 \mathrm{mg}$ treatment versus those treated with placebo. ${ }^{13}$

In the real-world study, 79/102 (77.5\%) patients had tried at least two unique triptans, and 51/102 (50.5\%) patients had tried at least three triptans. Previous triptans were ineffective for $61 / 86(70.9 \%)$ patients and associated with side effects for $14 / 86$ (16.3\%) patients. Although no specific post-hoc analysis was done on triptan insufficient responders, as most patients had tried at least 2 triptans, the overall efficacy appears encouraging. More real-world studies are needed to confirm the efficacy of ubrogepant in triptan insufficient responders.

\section{Coadministration of Ubrogepant with Other Medications}

Individualized migraine treatment includes potentially combining different types of acute migraine medications and often providing preventive therapies.

A Phase I, single-center, open-label, randomized, 3-way crossover, single-dose, pharmacokinetic interaction study investigated the pharmacokinetic interactions between sumatriptan and ubrogepant. In this study, 30 healthy participants received each of the following three medication regimens - single dose of ubrogepant $100 \mathrm{mg}$, single dose of sumatriptan $100 \mathrm{mg}$, and ubrogepant $100 \mathrm{mg}$ plus sumatriptan $100 \mathrm{mg}$, with a 7-day washout period between treatments. ${ }^{14}$ The study showed there were slight changes in the pharmacokinetic parameters of ubrogepant when administered with sumatriptan, in that the ubrogepant median time to maximum plasma concentration was delayed (3 hours [range: $1-5$ hours] vs 1.5 hours [range: $1-4$ hours]), mean maximum plasma concentration was reduced by $24 \%$ (coefficient of variation: 37.4\%) when ubrogepant was administered with sumatriptan $(n=29)$ compared with ubrogepant administered alone $(\mathrm{n}=30)$. The authors concluded such changes are expected to have minimal clinical relevance as no changes were seen in sumatriptan area under the curve and maximum plasma concentration when administered with ubrogepant. There was no treatmentemergent adverse event (TEAE) when ubrogepant was administered with sumatriptan. All adverse events in the study were mild and resolved within 24 hours. ${ }^{14}$

Since the gepants target CGRP, concerns exist regarding the efficacy and safety of concurrent use of gepants with other therapies that target CGRP such as the CGRP mAbs. To address this concern, an industry sponsored, multicenter, Phase Ib, open label study was conducted to evaluate the impact of erenumab or galcanezumab on the pharmacokinetics and safety of ubrogepant in 40 patients with migraine. The authors reported no significant changes in the pharmacokinetic parameters including ubrogepant peak plasma concentration and area under the plasma concentration-time curves after coadministration with erenumab versus being administered alone. The same conclusion was reached when ubrogepant was administered with galcanezumab. TEAEs when ubrogepant was given daily for 4 days following $\mathrm{mAb}$ were observed in $21.1 \%$ (galcanezumab) and $36.8 \%$ (erenumab) patients, including headache, constipation, abdominal discomfort, and nausea. No clinically relevant changes in laboratory parameters, vital signs, or electrocardiogram (ECG) values were observed. $^{15}$

In the real-world patient experience study done at a tertiary headache center, 62/106 (58.5\%) patients were concurrently using a CGRP mAb as migraine prevention. There was no difference in the "good responder" rates between those who were on a CGRP mAb versus those who were not on a CGRP mAb (41\% vs $56.8 \%, p=0.109)$. Good responder was defined as those who had headache relief within 2 hours after ubrogepant use for $\geq 75 \%$ of the treated attacks. As for safety evaluation, there was no difference on the overall adverse event rate between the two groups $(37.1 \%$ vs $43.2 \%, \mathrm{p}=0.528)$, although more patients rated the adverse events as moderate, as opposed to mild, in those who were concurrently using a CGRP $\mathrm{mAb}$ compared to those who were not $(47.8 \%$ vs $17.6 \%$, $p=0.048$ ). However, no patients rated the adverse events as severe regardless of concurrent use of a CGRP mAb.

\section{Adverse Events of Ubrogepant}

As for the safety evaluation, the most commonly reported adverse events within 48 hours of medication use in the ACHIEVE I and ACHIEVE II trials were nausea (1.7-$4.1 \%)$, somnolence $(0.6-2.1 \%)$, dry mouth (0.6-2.1\%), and dizziness (1.4-2.1\%). All the above adverse events 
were reported in less than $5 \%$ of the participants in each dosage group. ${ }^{1,2}$

A total of 2236 patients received ubrogepant in ACHIEVE I and ACHIEVE II. No serious adverse event was reported within 48 hours of ubrogepant use in both trials. Serious adverse events within 30 days were reported for 5 patients in ACHIEVE I (appendicitis, spontaneous abortion, pericardial effusion, and seizure) and for one participant in ACHIEVE II related to a bicycle accident. A total of 9 participants (5 in ACHIEVE I, 4 in ACHIEVE II) in the treatment group had post-baseline levels of ALT or AST that were at least 3 times the upper limit of the normal range; of which three cases ( 2 in ACHIEVE $I$ and 1 in ACHIEVE II) were judged to be possibly related to the trial regimen; however, the case in ACHIEVE II was randomized to placebo. ${ }^{1,2}$

The long-term safety and tolerability of repeated use of ubrogepant was further evaluated in the open-label, 52-week extension trial of ACHIEVE I and ACHIEVE II. After completing one of the two trials, a total of 1230 participants were re-randomized $1: 1: 1$ to usual care (417 patients), ubrogepant $50 \mathrm{mg}$ (404 patients), or ubrogepant $100 \mathrm{mg}$ (409 patients). A total of 233 (29\%) ubrogepant treated participants treated 6 or more migraine attacks during at least 1 month in the trial. Any adverse event occurred in 268 (66\%) participants, among them, approximately $10 \%$ were considered related to ubrogepant. The most reported TEAEs $(\geq 1 \%)$ in any ubrogepant dose group were nausea $(1.5 \%$ and $1.7 \%$ with ubrogepant 50 and $100 \mathrm{mg}$, respectively), dizziness $(0.5 \%$ and $1.5 \%)$, and somnolence $(1.5 \%$ and $1.2 \%)$. ALT increases $(0.7 \%$ and $1.0 \%)$ and AST increases $(0.5 \%$ and $1.0 \%)$ also occurred with ubrogepant 50 and $100 \mathrm{mg} .{ }^{11}$ The authors further analyzed the rates of TEAEs within $48 \mathrm{~h}$ post any dose of ubrogepant in various demographic and clinical characteristics subgroups, including age $<50$ vs age $\geq 50$, male vs female, white vs non-white, and body mass index $<30 \mathrm{vs} \geq 30$, and the presence of anxiety/depression or medication overuse, and reported that the safety and tolerability of ubrogepant were consistent within the various demographic and clinical characteristic subgroups. ${ }^{16}$ However, in the population analyzed, $90 \%$ were female and $85 \%$ were white. More studies are needed to confirm the findings in a more diverse population.

Adverse event rates were higher in the real-world study. The most common adverse events were fatigue in
$27.4 \%$, dry mouth in $7.5 \%$, nausea/vomiting $6.6 \%$, constipation in $4.7 \%$, and dizziness in $2.8 \%$. Possible explanations of the difference include that the most reported TEAEs from the clinical trials were listed in the real-world survey as a check-boxed design, the relatively small sample size of 106 patients, and that the majority of patients in the real-world study had chronic migraine and multiple previous ineffective and intolerance treatment trials. No serious adverse event was reported in the real-world study, and all adverse events resolved within 24 hours.

\section{Vascular Safety of Ubrogepant}

The vascular safety of CGRP targeted therapy, and the use in patients with high cardiovascular or cerebrovascular risk profiles have been areas of much discussion.

CGRP containing nerve fibers exist wildly in the cardiovascular system. Studies have shown that CGRP increases heart rate, cardiac contraction force, coronary blood flow, and microvascular permeability. CGRP is a potent vasodilator that plays a key role in regulating regional blood flow, auto-perfusion, and vascular resistance. ${ }^{17} \mathrm{~A}$ pre-clinical study showed that CGRP knockout mice had more extensive neuronal cell loss, higher levels of oxidative stress, and less compensatory capillary growth than wildtype in response to induced middle cerebral artery occlusion and bilateral common carotid artery stenosis. ${ }^{17}$ One in vitro study investigated the effects of ubrogepant and atogepant on CGRP-induced relaxation in human isolated middle meningeal, cerebral and coronary arteries. It showed that both ubrogepant and atogepant inhibited CGRP-induced relaxations more potently in intracranial arteries than coronary arteries, but neither of them demonstrated vasoconstrictive effect in coronary arteries. ${ }^{18}$

In the 52-week open-label extension of ACHIEVE I and ACHIEVE II, no cardiovascular TEAEs occurred at a rate $>2 \%$ in either ubrogepant treatment arm. One cardiovascular severe adverse event (sinus tachycardia) was considered to be related to ubrogepant. Of note, no events related to myocardial infarction or stroke were reported in either ubrogepant treatment arm. ${ }^{11}$ Two central nervous system severe adverse events were reported, both considered not related to ubrogepant.

Another pooled analysis based on the ACHIEVE I and ACHIVE II trials reported that $11 \%$ of participants were categorized as having a moderate-high cardiovascular risk $(\mathrm{n}=311), 32 \%$ a low cardiovascular risk $(\mathrm{n}=920)$, and $58 \%$ no cardiovascular risk factors $(\mathrm{n}=1670)$. The investigators 
reported that the proportion of ubrogepant participants reporting a TEAE was comparable across risk categories and did not differ greatly from placebo. ${ }^{19}$

Among the CGRP mAbs, erenumab is the only that targets the receptor. Of note, 61 cases of erenumabassociated elevated blood pressure have been reported in the post-market setting. ${ }^{20}$ Among them, 27 (44\%) cases required treatment for elevated blood pressure (including pharmacological intervention, emergency department visit, hospitalization). Nineteen (31\%) cases had pre-existing hypertension. ${ }^{20}$ Since gepants also target the CGRP receptor, concerns arise whether they could cause elevated blood pressure as well; however, gepants are currently being used as needed for the acute treatment of migraine and have short half-lives compared to CGRP monoclonal antibodies. Strict blood pressure monitoring was not included as part of the Phase III clinical trials or the realworld study of ubrogepant. Continued studies are needed to assess the cardiovascular safety of ubrogepant, especially with long-term and more frequent dosing paradigms that reflect real-world use of acute treatments for migraine as currently the safety of treating $>8$ migraines/month has not been established. ${ }^{8}$

\section{Ubrogepant and Medication Overuse Headache}

CGRP targeted therapy has been used for migraine prevention, including the CGRP monoclonal antibodies which are FDA approved for this indication and most recently rimegepant which was shown to be effective for migraine prevention when taken every other day but is not approved for migraine prevention at this time. ${ }^{21}$ If a therapy is working through pathways that prevent migraine, such as targeting CGRP, it would be assumed that they would have lower likelihood to cause medication overuse headache $(\mathrm{MOH})$. The question as to whether ubrogepant could cause medication overuse headache $(\mathrm{MOH})$, as seen with other acute medications such as triptans, has been raised. $^{22,23}$

Currently, there have not been human studies specifically on whether ubrogepant can cause MOH. A study used sumatriptan as a positive control and showed that ubrogepant and sumatriptan both demonstrated efficacy as acute medications for stress- and nitric oxide donorevoked cephalic allodynia in a preclinical model of $\mathrm{MOH}$, and that ubrogepant $100 \mathrm{mg} / \mathrm{kg}$ in repeated effective doses did not produce cutaneous allodynia or latent sensitization. ${ }^{24}$ More studies are needed to further evaluate whether ubrogepant can cause $\mathrm{MOH}$.

\section{Conclusion}

Ubrogepant is a CGRP receptor antagonist that received FDA approval for the acute treatment of migraine in adults. Phase III clinical trials, the 52-week open label extension, and a real-world study have demonstrated efficacy and safety of ubrogepant. Adverse events were more commonly reported in the real-world population. More studies are needed to evaluate the long-term efficacy and safety, especially the cardiovascular safety of ubrogepant and when combined with other CGRP modulating therapies.

\section{Abbreviations}

CGRP, calcitonin gene-related peptide; FDA, Food and Drug Administration; CT, calcitonin; AMY, amylin; AM, adrenomedullin; CLR, calcitonin receptor (CTR)-like receptor; $\mathrm{RAMP}_{1}$, receptor activity modifying protein 1 ; mAbs, monoclonal antibodies, CYP3A4, cytochrome P450 3A4; ICHD, International Classification of Headache Disorders; ALT, alanine aminotransferase; AST, aspartate aminotransferase; ECG, electrocardiogram; TEAEs, treatment-emergent adverse events; $\mathrm{MOH}$, medication overuse headache.

\section{Disclosure}

Dr Vander Pluym reports consulting for Teva and research grant from Amgen. The authors report no other conflicts of interest in this work.

\section{References}

1. Lipton RB, Dodick DW, Ailani J, et al. Effect of ubrogepant vs placebo on pain and the most bothersome associated symptom in the acute treatment of migraine: the ACHIEVE II randomized clinical trial. JAMA. 2019;322(19):1887. doi:10.1001/jama.2019.16711

2. Dodick DW, Lipton RB, Ailani J, et al. Ubrogepant for the treatment of migraine. N Engl J Med. 2019;381(23):2230-2241. doi:10.1056/ NEJMoa1813049

3. Moore E, Fraley ME, Bell IM, et al. Characterization of ubrogepant: a potent and selective antagonist of the human calcitonin generelated peptide receptor. $J$ Pharmacol Exp Ther. 2020; jpet.119.261065. doi:10.1124/jpet.119.261065

4. Yuan H, Spare NM, Silberstein SD. Targeting CGRP for the prevention of migraine and cluster headache: a narrative review. Headache J Head Face Pain. 2019;59(S2):20-32. doi:10.1111/head.13583

5. Croop R, Goadsby PJ, Stock DA, et al. Efficacy, safety, and tolerability of rimegepant orally disintegrating tablet for the acute treatment of migraine: a randomised, phase 3, double-blind, placebo-controlled trial. Lancet Lond Engl. 2019;394:737-745. doi:10.1016/S0140-6736(19)31606-X 
6. Chiang -C-C, Schwedt TJ. Calcitonin gene-related peptide (CGRP)targeted therapies as preventive and acute treatments for migraine the monoclonal antibodies and gepants. Prog Brain Res. 2020;255:143-170.

7. Amgen Inc.. Ubrelvy [package insert]. 2019.

8. Lexicomp. Ubrogepant: drug information. UpToDate, Waltham, MA. 2020.

9. Amgen Inc.. Aimovig [package insert]. Undefined. 2020.

10. Chiang C, Arca KN, Dunn RB, et al. Real-world efficacy, tolerability, and safety of ubrogepant. Headache J Head Face Pain. 2021. doi:10.1111/head.14062

11. Ailani J, Lipton RB, Hutchinson S, et al. Long-term safety evaluation of ubrogepant for the acute treatment of migraine: phase 3, randomized, 52-week extension trial. Headache J Head Face Pain. 2020;60(1):141-152. doi:10.1111/head.13682

12. Lipton R, Dodick D, Goadsby P, et al. Ubrogepant is effective in the acute treatment of migraine with mild pain. Neurology. 2020;94(15 Suppl):4726.

13. Lipton R, Singh RH, Revicki D, et al. Improved functionality, satisfaction, and global impression of change with ubrogepant for the acute treatment of migraine in triptan insufficient responders. In: 62nd American Headache Society Scientific meeting; NJ, USA: WILEY; 2020.

14. Jakate A, Boinpally R, Butler M, Lu K, McGeeney D, Periclou A. Evaluation of the pharmacokinetic interaction of ubrogepant coadministered with sumatriptan and of the safety of ubrogepant with triptans. Headache J Head Face Pain. 2020;60(7):1340-1350. doi:10.1111/head.13862

15. Jakate A, Boinpally R, Butler M, et al. Safety and tolerability of ubrogepant for the acute treatment of migraine following co-administration with preventive monoclonal antibody treatment. In: 62nd American Headache Society Scientific meeting; NJ, USA: WILEY; 2020.

16. Ailani J, Klein B, Mechtler L, et al. Safety and tolerability of ubrogepant within various demographic and clinical characteristic subgroups. In: 62nd American Headache Society Scientific meeting; NJ, USA: WILEY; 2020.
17. Zhai L, Sakurai T, Kamiyoshi A, et al. Endogenous calcitonin gene-related peptide suppresses ischemic brain injuries and progression of cognitive decline. J Hypertens. 2018;36(4):876-891. doi:10.1097/HJH.0000000000001649

18. Rubio-Beltran E, Chan KY, Danser AJ, MaassenVanDenBrink A, Edvinsson L. Characterisation of the calcitonin gene-related peptide receptor antagonists ubrogepant and atogepant in human isolated coronary, cerebral and middle meningeal arteries. Cephalalgia. 2019;40(4):357-366. doi:10.1177/0333102419884943

19. Severt L, Silberstein SD, Blumenfeld AM, et al. Safety and efficacy of ubrogepant in participants with moderate to high cardiovascular risk. Neurology. 2020;94(15Supplement):107.

20. Saely S, Croteau D, Jawidzik L, Brinker A, Kortepeter C. Hypertension: a new safety risk for patients treated with erenumab. Headache J Head Face Pain. 2021;61:202-208. doi:10.1111/ head.14051

21. Croop R, Lipton RB, Kudrow D, et al. Oral rimegepant for preventive treatment of migraine: a phase $2 / 3$, randomised, double-blind, placebo-controlled trial. Lancet. 2021;397(10268):51-60. doi:10.1016/S0140-6736(20)32544-7

22. Chiang -C-C, Schwedt TJ, Wang S-J, Dodick DW. Treatment of medication-overuse headache: a systematic review. Cephalalgia Int $J$ Headache. 2015;36(4):371-386. doi:10.1177/0333102415593088

23. Hagen K, Albretsen C, Vilming ST, et al. A 4-year follow-up of patients with medication-overuse headache previously included in a randomized multicentre study. $J$ Headache Pain. 2011;12 (3):315-322. doi:10.1007/s10194-010-0285-1

24. Navratilova E, Behravesh S, Oyarzo J, Dodick DW, Banerjee P, Porreca F. Ubrogepant does not induce latent sensitization in a preclinical model of medication overuse headache. Cephalalgia. 2020;40(9):892-902. doi:10.1177/0333102420938652
Journal of Pain Research

\section{Publish your work in this journal}

The Journal of Pain Research is an international, peer reviewed, open access, online journal that welcomes laboratory and clinical findings in the fields of pain research and the prevention and management of pain. Original research, reviews, symposium reports, hypothesis formation and commentaries are all considered for publication. The manuscript management system is completely online and includes a very quick and fair peer-review system, which is all easy to use. Visit http:// www.dovepress.com/testimonials.php to read real quotes from published authors. 